\title{
Effect of Aging on the Blowout Time in Various Ocular Vessels
}

\author{
Miyaji $\mathbf{A}^{1}$, Ikemura $\mathrm{T}^{2}$ and Hayashi $\mathbf{N}^{1^{*}}$ \\ ${ }^{1}$ Graduate School of Decision Science and Technology, Tokyo Institute of Technology, Meguro, Tokyo 152-8852, Japan \\ ${ }^{2}$ Faculty of Sport Sciences, Waseda University, Tokorozawa, Saitama 359-1192, Japan
}

*Corresponding author: Naoyuki Hayashi, Graduate School of Decision Science and Technology, Tokyo Institute of Technology, Meguro, Tokyo 152-8852, Japan, Tel: +81-3-57343434; Fax: +81-3-57343434; E-mail: naohayashi@hum.titech.ac.jp

Received date: February 17, 2016; Accepted date: March 28, 2016; Published date: March 31, 2016

Copyright: ( 2016 Miyaji A, et al. This is an open-access article distributed under the terms of the Creative Commons Attribution License, which permits unrestricted use, distribution, and reproduction in any medium, provided the original author and source are credited.

\begin{abstract}
The blowout time (BOT) is an index for assessing vascular function based on pulse wave analysis using laser speckle flowgraphy (LSFG). The BOT in the optic nerve head (ONH) is reportedly correlated with age. The ONH contains various vessels with differing properties, and it is unclear whether aging affects these vessels at similar rates. With the aim of using the BOT for the early detection of vascular changes, this study investigated whether there are regional differences in the effect of aging on the BOTs among the vessel area (MV), tissue area (MT), and all areas (MA) of the entire $\mathrm{ONH}$, retinal artery (RA), retinal and choroidal vessels (RCV), and retinal vein (RV). We measured the ocular blood flow velocity for $6 \mathrm{~s}$ in 14 young and 14 middle-aged males (20 \pm 2 years and $51 \pm 10$ years) three times using LSFG. The BOTs in the MV, MT, MA, RA, RCV, and RV were determined separately as the full width at half maximum of the mean blue rate in a heartbeat by pulse wave analysis. The BOTs in all of the targeted areas were significantly smaller in the middle-aged group than the young group and were significantly correlated with age. There was no significant interaction of age and differences in area. There were significant correlations in the BOTs between the MA and other targeted areas. It is suggested that the BOT in the entire ONH reflects the age-related changes in BOTs over a wide range of ocular vessels.
\end{abstract}

Keywords: Blowout time; Age; Optic nerve head; Retinal vessels; Choroidal vessels

\section{Introduction}

Aging increases the risk of visual impairment. According to global data on visual impairments reported in 2010 by the World Health Organization, the proportion of the population that is blind or visually impaired is sevenfold higher among those older than 50 years $(16.9 \%)$ than among those aged 15-49 years (2.4\%). The main causative diseases are cataract, glaucoma, and age-related macular degeneration, which are partially influenced by age-related vascular dysfunction [1-7]. Humans are highly dependent on visual information, and so the quality of life (QOL) can be expected to be markedly reduced by agerelated vascular dysfunction. Vascular aging is inevitable $[8,9]$, and so the early detection of ocular vascular dysfunction is necessary for preventing deterioration of the QOL.

Laser speckle flowgraphy (LSFG) noninvasively provides real-time, two-dimensional relative blood flow velocities in the ocular microcirculation based on the laser-speckle phenomenon, as described previously [10-15]. LSFG measures the mean blur rate (MBR), which reflects the relative blood flow velocity and is correlated with the actual blood flow volume as measured by hydrogen gas clearance and microsphere methods [16,17]. In addition, new software available for use with LSFG allows the analysis of the MBR pulse waveform [18-20]. The indexes calculated by this analysis can be compared between different individuals and between different time points. Several studies have developed indexes for estimating the condition of the vessels using LSFG.

The blowout time (BOT) is one of the indexes calculated by MBR pulse wave analysis using LSFG. This index is determined as the rate of the full width at half maximum of the MBR values in a heartbeat (see the Methods for details). A large BOT indicates that the vessels allow a large blood flow volume over a long time for a given blood pressure in each heartbeat, and in this condition the downstream tissues receive a good blood supply [15]. Some studies have found that the BOT in the optic nerve head $(\mathrm{ONH})$ is negatively correlated with age, suggesting that the BOT in the $\mathrm{ONH}$ is related to atherosclerotic changes and organ damage such as kidney disease [18-22].

Only the entire $\mathrm{ONH}$ circulation has been considered in previous studies observing the BOT, whereas LSFG allows quantification of the microcirculation separately in the $\mathrm{ONH}$, choroid, retinal vessels, separately. The information obtained from the $\mathrm{ONH}$ circulation as observed by LSFG is related to both retinal arterioles and venules, and choroidal capillaries. These vessels have different properties, such as in terms of the vascular diameter, types of constituent cells, and downstream tissues $[23,24]$. These differing properties could results in the vasculature in different regions being affected differently by aging.

It is still unclear whether aging affects on retinal and choroidal vessels at similar rates. Regarding the choroidal circulation, previous studies found that the blood flow volume but not the blood flow velocity decreases with aging due to decreases in the capillary density and diameter [25-27]. The effect of aging on the retinal circulation is controversial. Some studies found that the blood flow velocity in retinal artery decreases with age [28-30], whereas others found that the blood flow velocity either increases or is not correlated with age $[31,32]$. Tsuda et al. showed that the BOTs in the choroidal area and the entire area of the $\mathrm{ONH}$ were correlated with age, with the correlation being stronger in the former area [22]. Thus, it is likely that aging exerts different effects on the retinal and choroidal vessels. However, no previous study has separately assessed the retinal arterioles and venules. 
We hypothesized that there are regional differences in the effect of age on vascular changes even within a single eye. With the aim of early detection of vascular changes based on the BOT, this study investigated whether there are regional differences in the effect of aging on the BOTs among the entire $\mathrm{ONH}$, choroidal vessels, retinal artery, and retinal vein in healthy subjects covering a wide range of ages. Finding a similar rate of changes in the BOT among various target areas could lead to ONH observations being useful for estimating the effect of aging.

\section{Materials and Methods}

Fourteen young males [aged $20 \pm 2$ years (mean \pm SD) ranging from 18 to 26 years] and 14 middle-aged males [aged $51 \pm 10$ years ranging from 37 to 68 years] participated in this study. All of the subjects were free of any known autonomic dysfunction and cardiovascular and ocular diseases, and were not taking any medications. This study was approved by the ethics committee of the Tokyo Institute of Technology, Japan. All of the protocols used conformed with the standards set by the Declaration of Helsinki. Each subject received verbal and written explanations of the objectives, measurement techniques, and risks and benefits associated with the study, and then provided written informed consent.

After each subject had rested in a seated position for $30 \mathrm{~min}$, the blood pressure and MBR pulse waveform in the optic circulation were measured in an air-conditioned room maintained at $24^{\circ} \mathrm{C}$. Subjects were asked to open their right eye for $6 \mathrm{~s}$ while the laser-speckle image was recorded, which was repeated three times. They were requested to abstain from caffeinated beverages and strenuous exercise for $6 \mathrm{~h}$, and from eating for at least $2 \mathrm{~h}$ prior to the measurements.

The beat-by-beat arterial pressure was monitored continuously with an automatic sphygmomanometer whose probe was attached to the right middle finger (Finometer, Finapres Medical Systems, Amsterdam, The Netherlands), and it was transformed into the mean arterial pressure (MAP). The ocular circulation was then observed using LSFG (SoftCare, Fukuoka, Japan).

\section{Pulse wave analysis of the ocular circulation using LSFG}

The ocular circulation was observed from laser-speckle images using LSFG without the instillation of mydriatic drops. The laser wavelength was $830 \mathrm{~nm}$, and the observation field was 750 pixels $\times 360$ pixels, which corresponded to an ocular area of approximately $5 \mathrm{~mm} \times$ $3 \mathrm{~mm}$. The real-time blood flow map was obtained from the measured MBR (recorded at $30 \mathrm{frames} / \mathrm{s}$ ). The accompanying analysis software (LSFG analyzer, version 3.1.44.0) allows analysis of the MBR pulse waveform which corresponded to each cardiac cycle.

The MBR data were calculated from the following target areas, which are indicated by black circles and rectangles in Figure 1a: $\mathrm{ONH}$, retinal artery, retinal vein, and choroidal vessels. The LSFG software was used to automatically segment out vessels from the ONH based on a definitive threshold [33], and the mean MBRs were separately analyzed throughout the vessel (MV), tissue (MT) and all areas (MA) $[15,22]$. The MBRs in the retinal artery (RA) and retinal vein (RV) outside the $\mathrm{ONH}$ were calculated from the integral of a cross-sectional map of the MBRs within the selected arteriole and venules. The observation field marked by circle no. 3 in Figure 1a indicates the retinal and choroidal vessels (RCV) outside the ONH. The RCV data reflect not only the choroidal circulation but also the retinal circulation, since the LSFG cannot separate these two signals. In the present study, the measured MBR in RCV reflects mainly a change in the choroidal circulation, since choroidal vessels supply $85 \%$ of the total ocular blood flow to the retina [34].
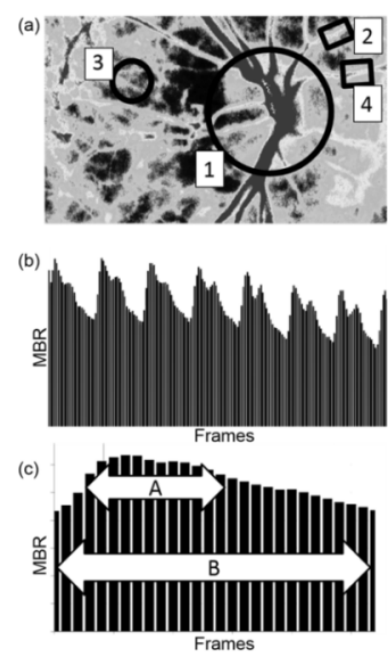

Figure 1: (a) An image of inner ocular circulation obtained using laser speckle flowgraphy. The actual obtained image was in color. Black circles and rectangles denote the regions of interest: (1) optic nerve head, (2) retinal artery, (3) retinal and choroidal vessels, (4) retinal vein. (b) Pulse waves calculated from each targeted vessel showing the change in the mean blur rate for 6s. (c) Continuous pulse waves in a targeted vessel shown in (b) are superimposed onto a single pulse wave. To calculate the blowout time (BOT), the number of frames in which the amplitude exceeded half the full width at half maximum of the mean blue rate $(\mathrm{A})$ was divided by the number of frames of a single normalized pulse (B).

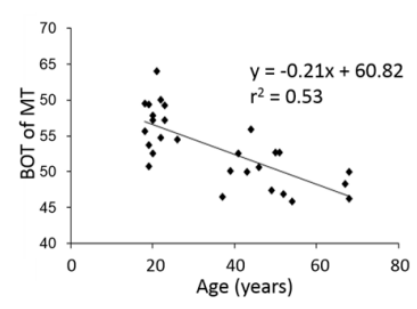

Figure 2: Relationship between aging and blowout times (BOTs) in the mean tissue area of the optic nerve head (MT). In all targeted areas, as well as the MT shown in this figure, the BOTs were significantly correlated with age.

The BOTs were obtained by MBR pulse waveform analysis using the LSFG analysis software. The measured MBR pulse waveforms corresponding to each cardiac cycle (Figure $1 \mathrm{~b}$ ) were normalized and superimposed onto a single pulse (Figure 1c). The number of frames in which the amplitude exceeded half the difference between the peak and valley of MBR values (A in Figure 1c) was divided by the total number of frames of the single pulse of the MBR (B in Figure 1c) [18-20]. Each BOT value was calculated automatically based on the following formula [18-20]: $\mathrm{BOT}=(\mathrm{A}) /(\mathrm{B}) \times 100$. We calculated the BOT 
Page 3 of 6

for three repeated observations and used the mean value in the subsequent analysis.

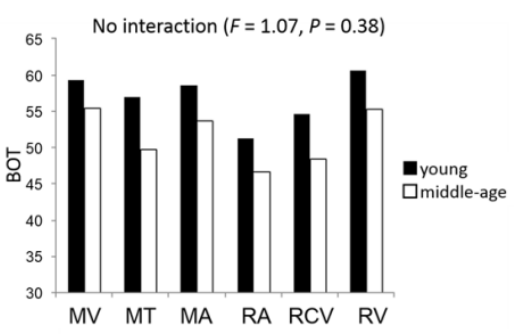

Figure 3: Mean BOT in various ocular vessels in the young and middle-aged groups. There were significant effects of aging and areas (both $\mathrm{P}<0.05$ ), but not interaction of age and differences in area. MV, mean value vessel area of the optic nerve head $(\mathrm{ONH})$. MT, mean value in the tissue area of the ONH. MA, mean value in all areas of the ONH. RA, retinal artery. RCV, retinal and choroidal vessels. RV, retinal vein.

\section{Data analysis}

The data are expressed as mean and SE values. The cutoff for statistical significance was set at $\mathrm{P}<0.05$. Pearson correlation analysis was used to evaluate the relationship between age and the BOTs in each area. The effects of age and areas on the measured BOTs were tested using two-way repeated ANOVA. When a significant $F$ value was detected, this was further examined using Bonferroni's post hoc test to assess for the effect of areas, and using the unpaired t-test to compare the BOT values in each area between the young and middleaged groups. All statistical analyses were performed with the Statistical Package for the Social Sciences (IBM SPSS Statistics 21.0 for Windows, IBM, Tokyo, Japan).

\section{Results}

The physical characteristics in the young and middle-aged groups are listed in Table 1. The height and body mass did not differ significantly between the two groups, whole the MAP was significantly larger in the middle-aged group than the young group.

\begin{tabular}{|c|c|c|}
\hline & Young group & Middle-aged group \\
\hline Age (years) & $21 \pm 2$ & $51 \pm 10^{*}$ \\
\hline Height $(\mathrm{cm})$ & $170.1 \pm 6.4$ & $171.6 \pm 4.3$ \\
\hline Body mass $(\mathrm{kg})$ & $60.2 \pm 8.5$ & $65.8 \pm 8.3$ \\
\hline MAP $(\mathrm{mmHg})$ & $81 \pm 8$ & $96 \pm 13^{*}$ \\
\hline
\end{tabular}

Table 1: Characteristics of the subjects in the young and middle-aged groups. Data are mean and SE values. Age and mean arterial pressure (MAP) differed significantly between the two groups. ${ }^{*} \mathrm{P}<0.05$ young vs. middle-aged groups.

In both groups, the BOT was significantly larger in the MV than in the MT, and significantly smaller in the RA than in the RV. There was no significant change in the BOT between the MT and RCV in both groups. The BOT in all of the targeted areas were significantly smaller in the middle-aged group than the young group (Table 2), and significantly correlated with age for all subjects (Table 3 and Figure 2). There was no significant interaction of age and differences in area $(\mathrm{F}=1.07, \mathrm{P}=0.38$ ) (Figure 3 ). In addition, there were significant correlations in the BOTs between the MA of the $\mathrm{ONH}$ and other targeted areas (Table 4).

\begin{tabular}{|c|c|c|}
\hline & Young group & Middle-aged group \\
\hline BOT MV & $59.2 \pm 0.8$ & $55.5 \pm 0.8^{*}$ \\
\hline MT & $56.9 \pm 0.9$ & $49.8 \pm 0.8^{*}$ \\
\hline MA & $58.5 \pm 0.8$ & $53.7 \pm 0.8^{*}$ \\
\hline RA & $51.2 \pm 0.9$ & $46.7 \pm 0.8^{*}$ \\
\hline RCV & $54.7 \pm 1.3$ & $48.4 \pm 0.8^{*}$ \\
\hline RV & $60.6 \pm 1.7$ & $55.3 \pm 1.4^{*}$ \\
\hline
\end{tabular}

Table 2: Comparison of the blowout time (BOT) in each targeted area between the young and middle-aged groups. The BOT was significantly larger in the young group than the middle-aged group in all targeted areas. MV, mean value in the vessel area of the optic nerve head (ONH). MT, mean value in the tissue area of the ONH. MA, mean value in all areas of the ONH. RA, retinal artery. RCV, retinal and choroidal vessels. $\mathrm{RV}$, retinal vein. ${ }^{*} \mathrm{P}<0.05$ young vs. middle-aged groups.

\begin{tabular}{|c|c|c|}
\hline & $r$ & $P$ \\
\hline MV & -0.59 & $<0.05$ \\
\hline MT & -0.73 & $<0.05$ \\
\hline MA & -0.65 & $<0.05$ \\
\hline RA & -0.49 & $<0.05$ \\
\hline RCV & -0.59 & $<0.05$ \\
\hline RV & -0.38 & $<0.05$ \\
\hline
\end{tabular}

Table 3: Relationship between age and blowout times in each targeted area. In all targeted areas, the blowout times were significantly correlated with age. MV, mean value vessel area of the optic nerve head $(\mathrm{ONH}) . \mathrm{MT}$, mean value in the tissue area of the ONH. MA, mean value in all areas of the ONH. RA, retinal artery. RCV, retinal and choroidal vessels. $\mathrm{RV}$, retinal vein.

\begin{tabular}{|c|c|c|}
\hline & $r$ & $P$ \\
\hline MV & 0.97 & $<0.05$ \\
\hline MT & 0.92 & $<0.05$ \\
\hline RA & 0.40 & $<0.05$ \\
\hline RCV & 0.75 & $<0.05$ \\
\hline RV & 0.48 & $<0.05$ \\
\hline
\end{tabular}

Table 4: Relationships in the blowout times between the mean all areas of the optic nerve head and other targeted areas. There were significant correlations in the blowout times between the mean all areas of the optic nerve head (ONH) and other targeted areas, respectively. MT, 
mean value in the tissue area of the ONH. MA, mean value in all areas of the ONH. RA, retinal artery. RCV, retinal and choroidal vessels. RV, retinal vein.

\section{Discussion}

The present study investigated the effect of aging on the BOTs in the following vessels and tissue areas: MV, MT, MA, RA, RCV, and RV. The main findings of the present study were as follows. First, the BOTs in all of the targeted areas were negatively correlated with age, even though these areas include arteries, veins, and capillaries with marked differences in properties. Second, there was no significant interaction of age and differences in area on BOTs. Finally, the BOT in the MA of the $\mathrm{ONH}$, which has been frequently used to assess the functions of ocular vessels, was significantly and positively correlated with BOTs in other targeted areas, even though the BOT values differed among the areas. These observations suggest that the BOT in the entire ONH reflects age-related changes in the BOTs over a wide range of ocular vessels.

The BOT in the entire $\mathrm{ONH}$ as calculated by MBR pulse wave analysis with LSFG is frequently used, and is a candidate predictor for age-related vascular changes. This index makes it possible to investigate differences among individuals and the effects of aging, although the blood flow itself is a relative value and so cannot be used for comparing between different subjects. The ONH is the landmark for ocular observations since it is easy to identify the same site in each individual. In addition, it has been reported that the reproducibility of the MBR in the entire ONH is slightly better than those of the RA, RV, and choroidal vessels outside the ONH [35]. Thus, many studies investigating the BOT have focused on the entire $\mathrm{ONH}$.

The BOT in the entire $\mathrm{ONH}$ was correlated with aging and indexes of BOTs in other areas. The reliability of using the BOT in the entire $\mathrm{ONH}$ for assessing ocular vessels has been controversial [18-22], since the entire $\mathrm{ONH}$ includes a mixture of choroid and retinal vessels that have different properties, such as in terms of the vascular diameter, innervations, types of constituent cells, and downstream tissues $[23,24]$. The rate of aging in these vessels could differ, and thus which vessels are affected by aging remains to be elucidated. In the present study, the BOTs in the vessel area and tissue areas of the ONH, and the $\mathrm{RA}, \mathrm{RCV}$, and $\mathrm{RV}$ which exist outside the $\mathrm{ONH}$ area all showed negative relationships with age, as well as all areas of the entire ONH. In addition, the BOT in the entire $\mathrm{ONH}$ was significantly related to the BOTs in the other targeted areas. Thus, the BOT in the entire ONH can reflect the age-related changes in the ocular circulation that involve mixtures of various vessel types.

The BOTs in all of the targeted areas were significantly smaller in the middle-aged group than the young group, and there were significant correlations in the BOTs in all of the targeted areas with age. There was an effect of aging on the BOTs in widespread areas of ocular vessels. Most previous studies used the entire $\mathrm{ONH}$ when confirming that the BOT of the ONH is correlated with age [18-22]. Only one research group has assessed the BOTs in separate tissue areas of the $\mathrm{ONH}$ and found that the tissue area of the $\mathrm{ONH}$ was correlated with aging [22]. However, no previous study has assessed the effect of age on the BOTs in large vessels such as RAs and RVs inside the ONH and in areas outside the ONH. The BOTs in various areas of the ocular circulation decrease with aging, regardless of the type of vasculature.

The following effects of area differences among BOTs were found: MV vs. MT and MT vs. MA inside the ONH, and RA vs. RV outside the ONH. The BOT in the RV outside the ONH was significantly larger than that in the RA, possibly due to the difference between capacitance and resistance vessels. These results suggest that there are regional differences in the BOTs among various ocular areas.

There was no interaction of age and differences in area, which did not support our hypothesis. We hypothesized that aging exerts different effects on various ocular vessels, because the vascular properties differ considerably between the RA, RV, and choroidal vessels $[23,24]$, and the correlation with aging in the BOT in choroidal vessels inside the $\mathrm{ONH}$ was stronger than that in the entire $\mathrm{ONH}$ [22]. No interaction of age and area was found in the BOTs in the present study. Thus, the BOTs in all of the targeted areas decrease with age at a similar rate, and there are no regional differences in the effect of aging on changes in BOT.

In the present study, the coefficient of variation for the BOT was higher in the $\mathrm{ONH}$ than outside the $\mathrm{ONH}$ as calculated from three repetitions of measurements $(3.7,3.1,3.4,5.2,4.2$, and 7.4 in the MV, MT, MA, RA, RCV, and RV, respectively). Aizawa et al. reported that the coefficient of variation for MBR measurements in vessels such as RAs and RVs was worse than those in the entire ONH and RCV, due to the small region of interest $(1.9,2.1,2.9,8.7,4.1$, and 6.2 in the MV, MT, MA, RA, RCV, and RV, respectively) [35]. The different relationship with age between the choroidal circulation and the entire ONH reported previously [22] might have been due to the different reproducibilities of the MBR in these areas; that is, the differences reflect methodological limitations rather than actual regional differences. The reproducibility in each area in the present study was similar to that found previously [35], even though the coefficient of variation was calculated in the present study from only three repetitions. This high reproducibility of the BOT in the entire ONH in both the previous and present studies suggests that the $\mathrm{ONH}$ is an appropriate area for assessing age-related vascular changes in the ocular circulation.

The BOT is calculated based on the number of frames, and the frame rate was 30 frames/s in this study. Thus, supposing a resting heat rate of $60 \mathrm{bpm}$, the time resolution in the present method was $3.3 \mathrm{for}$ the BOT. The step size is less than 3.3 in Figure 2 due to the three observations being averaged. This value exceeded the difference obtained from aging; however, it should be noted that the original time resolution was not large compared to the BOT values.

Previous studies have suggested that the BOT in the $\mathrm{ONH}$ is correlated with not only age but also the carotid intima-media thickness and brachial-ankle pulse wave velocity [18-22]. The eye is the only window allowing the noninvasive and direct observation of vessels, and thus the BOT is an appropriate tool for comparing vascular function among individuals. Individual differences in the geometric vascular pathway in the $\mathrm{ONH}$ are smaller than those in the downstream areas of the ONH $[24,36]$. The $\mathrm{ONH}$ is the landmark of the observation of the ocular circulation. Thus, observations of the vasculature in the $\mathrm{ONH}$ can be used for a practical index of the BOT. The results of the present study support the reliability of the BOT of the entire $\mathrm{ONH}$ being representative of changes in the ocular circulation with aging; however, further longitudinal studies investigating the relationship between the BOT and age are needed. Also, the reliability of assessing the aging of systemic vasculature by using the BOT in the $\mathrm{ONH}$ is another issue that should be addressed in the future. 


\section{Conclusion}

This study investigated whether there are regional differences in age-related changes in different vasculatures in the ocular circulation by comparing BOTs which is an index of vascular function in the MV, MT, MA, RA, RCV, and RV between the subjects in the young and middle-aged groups. There was an effect of age on the BOTs in all targeted areas of the ocular circulation. There were also effects of differences in the areas of measured BOTs in both groups. However, there was no interaction of age and differences in area. In addition, the BOT in the entire $\mathrm{ONH}$, which is an often-used area for observing ocular circulation, reflected the age-related changes in the BOTs in other targeted areas. These findings indicate that there are no regional differences in the age-related vascular changes in the ocular circulation, and that the BOT derived only for the entire $\mathrm{ONH}$ can be used to assess age-related vascular changes in most of the ocular circulation.

\section{Acknowledgement}

This work was supported by JSPS KAKENHI grants: number 15J11556 to A.M. and number 26560396 to N.H. The authors have no financial conflict of interest.

\section{Declaration of interest}

All authors have no conflict of interest.

\section{References}

1. Bertelsen M, Linneberg A, Rosenberg T, Christoffersen N, Vorum H, et al. (2012) Comorbidity in patients with branch retinal vein occlusion: case-control study. BMJ 345: e7885.

2. Caprioli, Coleman AL; Blood Flow in Glaucoma Discussion (2010) Blood pressure, perfusion pressure, and glaucoma. Am J Ophthalmol 149: 704-712.

3. Rehak J, Rehak M (2008) Branch retinal vein occlusion: pathogenesis, visual prognosis, and treatment modalities. Curr Eye Res 33: 111-131.

4. Ryskulova, Turczyn K, Makuc DM, Cotch MF, Klein RJ, et al. (2008) Selfreported age-related eye diseases and visual impairment in the United States: results of the 2002 national health interview survey. Am J Public Health 98: 454-461.

5. Venkataraman, Flanagan JG, Hudson C (2010) Vascular reactivity of optic nerve head and retinal blood vessels in glaucoma--a review. Microcirculation 17: 568-581.

6. Werne, Harris A, Moore D, BenZion I, Siesky B (2008) The circadian variations in systemic blood pressure, ocular perfusion pressure, and ocular blood flow: risk factors for glaucoma? Surv Ophthalmol 53: 559-567.

7. World Health Organization. Global data on visual impairments 2010.

8. Olser W (1898) The Principles and Practice of Medicine. Appleton, New York.

9. Seals, Desouza CA, Donato AJ, Tanaka H (2008) Habitual exercise and arterial aging. J Appl Physiol (1985) 105: 1323-1332.

10. Tamaki Y, Araie M, Kawamoto E, Eguchi S, Fujii H (1994) Noncontact, two-dimensional measurement of retinal microcirculation using laser speckle phenomenon. Invest Ophthalmol Vis Sci 35: 3825-3834.

11. Tamaki, Araie M, Kawamoto E, Eguchi S, Fujii H (1995) Non-contact, two-dimensional measurement of tissue circulation in choroid and optic nerve head using laser speckle phenomenon. Exp Eye Res 60: 373-383.

12. Fujii H, Nohira K, Yamamoto Y, Ikawa H, Ohura T (1987) Evaluation of blood flow by laser speckle image sensing. Part 1. Appl Opt 26: 5321-5325.
13. Isono $\mathrm{H}$, Kishi S, Kimura $\mathrm{Y}$, Hagiwara N, Konishi N, et al. (2003) Observation of choroidal circulation using index of erythrocytic velocity. Arch Ophthalmol 121: 225-231.

14. Sugiyama, Araie M, Riva CE, Schmetterer L, Orgul S (2010) Use of laser speckle flowgraphy in ocular blood flow research. Acta Ophthalmol 88: 723-729.

15. Sugiyama T (2014) Basic technology and clinical applications of the updated model of laser speckle flowgraphy to ocular diseases. Photonics 1: 220-234.

16. Takahashi, Sugiyama T, Tokushige H, Maeno T, Nakazawa T, et al. (2013) Comparison of CCD-equipped laser speckle flowgraphy with hydrogen gas clearance method in the measurement of optic nerve head microcirculation in rabbits. Exp Eye Res 108: 10-15.

17. Wang L, Cull GA, Piper C, Burgoyne CF, Fortune B (2012) Anterior and posterior optic nerve head blood flow in nonhuman primate experimental glaucoma model measured by laser speckle imaging technique and microsphere method. Invest Ophthalmol Vis Sci 53: 8303-8309.

18. Shiba T, Takahashi M, Hori Y, Maeno T, Shirai K (2012) Optic nerve head circulation determined by pulse wave analysis is significantly correlated with cardio ankle vascular index, left ventricular diastolic function, and age. J Atheroscler Thromb 19: 999-1005.

19. Shiba T, Takahashi M, Hori Y, Maeno T (2012) Pulse-wave analysis of optic nerve head circulation is significantly correlated with brachial-ankle pulse-wave velocity, carotid intima-media thickness, and age. Graefes Arch Clin Exp Ophthalmol 250: 1275-1281.

20. Shiba T, Takahashi M, Maeno T (2014) Pulse-wave analysis of optic nerve head circulation is significantly correlated with kidney function in patients with and without chronic kidney disease. J Ophthalmol.

21. Shiga Y, Omodaka K, Kunikata H, Ryu M, Yokoyama Y, et al. (2013) Waveform analysis of ocular blood flow and the early detection of normal tension glaucoma. Invest Ophthalmol Vis Sci 54: 7699-7706.

22. Tsuda S, Kunikata H, Shimura M, Aizawa N, Omodaka K, et al. (2014) Pulse-waveform analysis of normal population using laser speckle flowgraphy. Curr Eye Res 39: 1207-1215.

23. Netter FH (2011) Atlas of Human Anatomy, 5th ed. Saunders, Philadelphia.

24. Amemiya T (2012) Retinal and Choroidal Vascular Changes and Systemic Diseases in Rats: Corrosion Cast and Scanning Electron Microscopy. Springer-Verlag Tokyo, Japan.

25. Ito YN, Mori K, Young-Duvall J, Yoneya S (2001) Aging changes of the choroidal dye filling pattern in indocyanine green angiography of normal subjects. Retina 21: 237-242.

26. Grunwald JE, Hariprasad SM, DuPont J (1998) Effect of aging on foveolar choroidal circulation. Arch Ophthalmol 116: 150-154.

27. Ramrattan RS, van der Schaft TL, Mooy CM, de Bruijn WC, Mulder PG, et al. (1994) Morphometric analysis of Bruch's membrane, the choriocapillaris, and the choroid in aging. Invest Ophthalmol Vis Sci 35: 2857-2864.

28. Yaoeda K, Shirakashi M, Funaki S, Funaki H, Nakatsue T, et al. (2000) Measurement of microcirculation in optic nerve head by laser speckle flowgraphy in normal volunteers. Am J Ophthalmol 130: 606-610.

29. Gillies WE, Brooks AM, Scott M, Ryan L (1999) Comparison of colour Doppler imaging of orbital vessels in elderly compared with young adult patients. Aust N Z J Ophthalmol 27: 173-175.

30. Groh MJ, Michelson G, Langhans MJ, Harazny J (1996) Influence of age on retinal and optic nerve head blood circulation. Ophthalmology 103: 529-534.

31. Harris A, Chung HS, Ciulla TA, Kagemann L (1999) Progress in measurement of ocular blood flow and relevance to our understanding of glaucoma and age-related macular degeneration. Prog Retin Eye Res 18: 669-687.

32. Williamson TH, Lowe GD, Baxter GM (1995) Influence of age, systemic blood pressure, smoking, and blood viscosity on orbital blood velocities. Br J Ophthalmol 79: 17-22. 
Citation: Miyaji A, Ikemura T, Hayashi N (2016) Effect of Aging on the Blowout Time in Various Ocular Vessels. Aging Sci 4: 148. doi: 10.4172/2329-8847.1000148

Page 6 of 6

33. Okamoto K, Thuy L, Takahashi N, Yasumoto A, Fujii H (2010) Analysis of blood flow in retinal vessels using laser speckle flowgraphy. Atarashii Ganka 27: 256-259.

34. Alm A, Bill A (1973) The effect of stimulation of the cervical sympathetic chain on retinal oxygen tension and on uveal, retinal and cerebral blood flow in cats. Acta Physiol Scand 88: 84-94.

35. Aizawa N, Yokoyama Y, Chiba N, Omodaka K, Yasuda M et al. (2011) Reproducibility of retinal circulation measurements obtained using laser speckle flowgraphy-NAVI in patients with glaucoma. Clin Ophthalmol 5: 1171-1176.

36. Grauslund J, Green A, Kawasaki R, Hodgson L, Sjølie AK, et al. (2010) Retinal vascular fractals and microvascular and macrovascular complications in type 1 diabetes. Ophthalmology 117: 1400-1405. 